\title{
Optical imaging of the effect of in-plane fields on cholesteric liquid crystals
}

\author{
S. A. Jewell and J. R. Sambles \\ Thin Film Photonics Group, School of Physics, University of Exeter, Stocker Road, Exeter, EX4 4QL, United Kingdom
}

(Received 15 April 2008; published 22 July 2008)

\begin{abstract}
The effects of in-plane electric fields on the director structure of cholesteric liquid crystals has been imaged in three dimensions using fluorescence confocal polarizing microscopy. The results show that a liquid crystal lying outside the electrode gap can be significantly affected by stray fields occurring above the electrode surface, resulting in a $90^{\circ}$ rotation of the cholesteric helix. Distinct differences between the behavior of cholesterics with positive and negative dielectric anisotropies are observed.
\end{abstract}

DOI: 10.1103/PhysRevE.78.012701

PACS number(s): 61.30.Gd, 42.70.Df

\section{INTRODUCTION}

The use of in-plane electric fields to control the liquid crystal alignment in flat-panel displays is becoming increasingly common [1]. In this structure the transmission of light through individual pixels is controlled by a potential difference applied between electrodes on the back plate of the liquid crystal display, separated by a distance of the order of micrometers parallel to the substrate. This electrode system is used to control the director (average liquid crystal alignment) in recently developed commercially significant liquid crystal structures such as in-plane switching (IPS) [1] and fringe-field switching (FFS) [2] displays, which offer advantages such as improved viewing angle and optical contrast when compared to traditional twisted nematic liquid crystal structures [3]. As the demand for increased resolution grows, along with miniaturization for projection-based liquid crystal displays, so the influence of these switching fields on neighboring pixels becomes increasingly problematic. Interestingly though, stray fields emanating from in-plane electrodes have been indirectly observed to contribute to the switching characteristics of FFS and IPS displays by considering viewing angle characteristics [2].

One method for producing three-dimensional (3D) images of the effects of the switching fields on liquid crystal alignments in situ is fluorescence confocal polarizing microscopy (FCPM) [4,5]. This technique involves doping the liquid crystal material with a low concentration $(\sim 0.01 \mathrm{wt} \%)$ of a high-quantum-yield fluorescent dye, which absorbs strongly at one wavelength and emits at one of a higher value. A suitably anisotropic dye molecule will align with the liquid crystal, following the director alignment in the cell. For a linearly polarized excitation beam, the efficiency of the light absorption of the dye and the strength of the fluorescence emission are controlled by the angle $\theta$ between the polarization axis of the incident light and the absorption axis of the dye molecule, with the fluorescence intensity produced being proportional to $\cos ^{2} \theta$. The dye (and hence director) orientation is imaged by mounting the sample in a confocal fluorescence microscopy setup [6] and scanning over a $\sim 100$ $\times 100 \mu \mathrm{m}^{2}$ area in the $x-y$ plane at a fixed depth $z$. An optical "slice" of intensity versus position is recorded, and by repeating this at other depths in the cell, a $3 \mathrm{D}$ model of the director orientation is produced. The use of a pinhole in the optical setup (Fig. 1) ensures that only light emitted from the focal region of the confocal beam is collected, allowing a spatial resolution of less than $1 \mu \mathrm{m}$ in all three dimensions.

\section{EXPERIMENT}

Several liquid crystal cells containing in-plane electrodes with a variety of electrode-gap sizes were constructed for study in a FCPM system. For the lower substrate, a 1.5 -mm-thick glass slide $(n=1.52)$ was used, onto which was deposited $100 \mathrm{~nm}$ of silver, using thermal evaporation, with a glass fiber used as a mask to create an electrode gap of the required width. The upper substrate was formed from a 160 - $\mu$ m-thick coverglass slip $(n=1.52)$ with both glass plates being spin coated with a polyimide (JSR AL1254), baked, and rubbed perpendicular to the electrode gap to produce homogeneous alignment. The cell was then assembled using 15- $\mu \mathrm{m}$-thick Mylar as spacers along two edges (Fig. 2). Two liquid crystals, ZLI-2293 (Merck KGaA, $\Delta n$ $=0.133, \Delta \varepsilon=+10)$ and MLC-6610 (Merck KGaA, $\Delta n$ $=0.098, \Delta \varepsilon=-3.1)$, were doped with the chiral dopant CB15 $(\sim 2.7 \mathrm{wt} \%)$ to create a cholesteric pitch of $\sim 5 \mu \mathrm{m}$ in each material. These host liquid crystals were selected because the parameter $p \Delta n / 2 \lambda$ in each case is small enough to reduce the influence of the Maugin effect on the resulting images [7]. To allow imaging from the FCPM system, each cholesteric material was doped with a low concentration $(0.01 \mathrm{wt} \%)$ of the

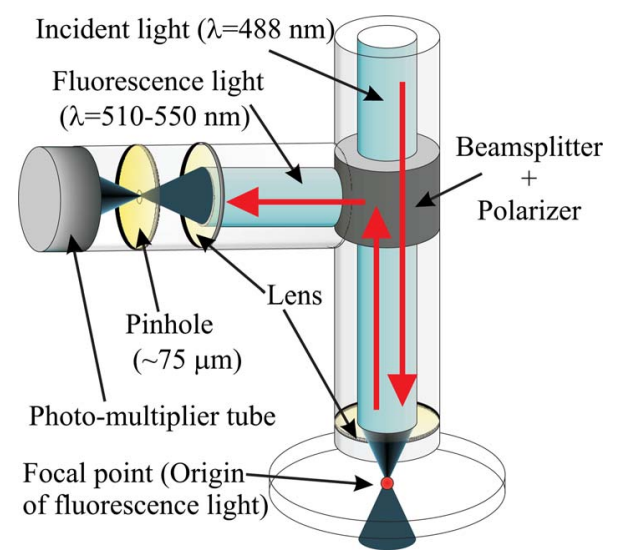

FIG. 1. (Color online) Schematic diagram of the arrangement of optical components in a fluorescence confocal polarizing microscopy system. 


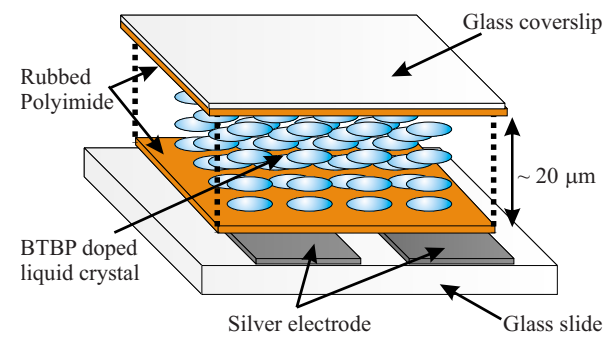

FIG. 2. (Color online) Schematic diagram of a typical in-plane switching homogeneously aligned liquid crystal cell for use in the FCPM imaging study.

fluorescent dye $N, N$-bis(2,5-di-tert-butylphenyl)-3,4,9,10perylenedicarboximide (BTBP) and allowed to mix in the isotropic phase overnight. The mixtures were then introduced into separate cells and examination under crossed polarizers suggested that in each case a good cholesteric monodomain was formed.

Each cell was mounted in turn in an inverted confocal fluorescence microscope system (Leica TCS SP5), operating in reflection mode, with the coverslip (incident face) indexmatched to a $63 \times$ oil immersion lens (numerical aperture 1.4) with the rubbing direction parallel to the polarization of the incident light. An excitation wavelength of $488 \mathrm{~nm}$ was used and fluorescence light in the range $510-550 \mathrm{~nm}$ was collected. FCPM images were obtained from a 120 $\times 30 \mu \mathrm{m}^{2}$ region in the $x-y$ plane in $0.2 \mu \mathrm{m}$ steps through the cell, spanning a total distance of $40 \mu \mathrm{m}$ in the $z$ direction. This was then repeated with various $10 \mathrm{kHz}$ ac potential differences applied across the electrode gap, with the sample left for 10 min prior to the scan commencing to allow the sample to reach equilibrium. For each set of data, image reconstruction software was used to compile the in-plane images and construct a cross section through the cell showing how the fluorescent intensity varies with depth.

\section{RESULTS AND DISCUSSION}

The reconstructed images of cross sections through the ZLI-2293 and MLC-6610 cells at various voltages are shown in Figs. 3 and 4 respectively. The images clearly indicate a cholesteric structure of pitch $\sim 5 \mu \mathrm{m}$ for both the positive and negative dielectrically anisotropic materials. This is evident from the dark and light bands in the cross sections collected at $0 \mathrm{~V}$ [Figs. 3(a) and 4(a)] indicating that the fluorescent dye (and hence the director) is periodically either parallel or perpendicular to the polarization of the excitation beam, resulting in maximum or minimum fluorescence, respectively. On application of a potential difference across the in-plane electrode gap, distinct differences are seen between the response of the positive and negative dielectrically anisotropic materials. When an in-plane field above a threshold voltage of $\sim 13 \mathrm{~V}_{p p}$ is applied across the $25 \mu \mathrm{m}$ electrode gap of the cell containing the positive material (ZLI-2293), a buckling of the cholesteric layers is initially observed outside the electrode gap [Fig. 3(b)]. In addition, the liquid crystal in immediate contact with the lower substrate (i.e., in the region of the strongest field) appears to untwist and align with the

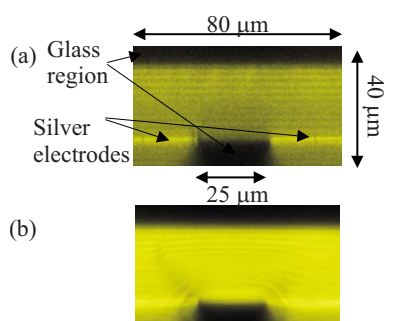

(c)

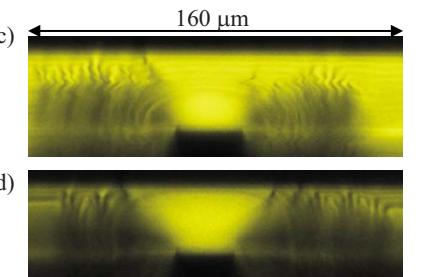

FIG. 3. (Color online) Cross-section images from a ZLI-2293 liquid crystal cell showing the fluorescence intensity as a function of position at applied peak-to-peak voltages of (a) 0 , (b) 15, (c) 25, and (d) $50 \mathrm{~V}$.

applied field, as predicted from consideration of weak azimuthal anchoring [8]. As the voltage is increased the proportion of the cell unwound by the field increases and, in addition, the reorientation of the liquid crystal outside the electrode gap becomes increasingly pronounced. In this region, the liquid crystal appears to form a cholesteric structure with the helix lying along the $x$ axis of the system with a $5 \mu \mathrm{m}$ pitch. The clarity of the pitch structure at the higher voltages is blurred due to the movements of defects through the region which mediate the helical reorientation while the data are collected.

When an in-plane field is applied to the cell containing the negative material (MLC-6610), the application of a voltage above a threshold potential of $\sim 30 \mathrm{~V}_{p p}$ across the $65 \mu \mathrm{m}$ gap initially causes a buckling of the cholesteric layers within the electrode gap [Fig. 4(b)]. Interestingly, the pitch of the cholesteric is maintained in the distorted structure, with the formation of these deformations mediated by defects observed to move along the $y$ axis, parallel to the electrode

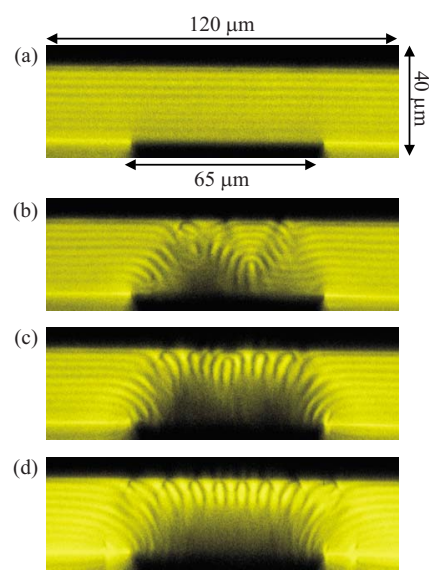

FIG. 4. (Color online) Cross-section images from a MLC-6608 liquid crystal cell showing the fluorescence intensity as a function of position at applied peak-to-peak voltages of (a) 0, (b) 40, (c) 60, and (d) $100 \mathrm{~V}$. 


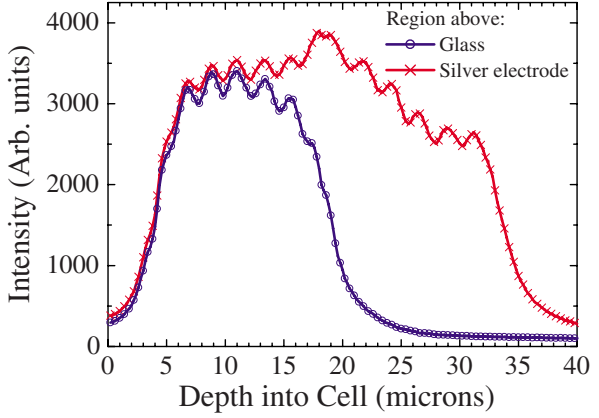

FIG. 5. (Color online) Comparison of recorded fluorescence intensity (arbitrary units) versus depth into the sample for regions above the silver electrode and glass electrode gap.

edges, over a period of $10 \mathrm{~min}$ or more. As the voltage is increased the liquid crystal within the electrode gap clearly forms a cholesteric structure with the helix axis lying across the electrode gap (along the $x$ axis) and hence the ordinary axis of the liquid crystal lies parallel to the electric field at all points in the gap. In this conformation both the dielectric and elastic (twist) energies of the system are minimized.

In all of these images, an interesting optical effect arises due to the use of metallic silver as the electrode material. The black area that can clearly be seen in the lower central region of each cross-sectional image is attributed to the presence of the glass substrate, which has no fluorescence at the wavelength of the excitation laser and therefore appears dark when scanned. However, in the adjacent glass regions lying below the silver electrode a spatially periodic high fluorescence intensity is recorded. This is due to the incident light, with a focal point below the silver surface, being reflected from the metal and focused at a point within the cell. Likewise, the fluorescence produced at this position is therefore also in focus when it reaches the pinhole in the FCPM system and so the fluorescence intensity is recorded as originating from the original (but false) focal point. Figure 5 shows a plot of the detected intensity versus focal depth (along the $z$ axis) for points in the $x-y$ plane above the silver electrode and above the glass in the electrode gap. The cholesteric pitch of the material can be clearly distinguished in both data sets with the signal collected from the light below the electrode region still showing a clear fluorescence variation. (The oscillations from the fluorescence variation attributed to the cholesteric structure of the liquid crystal appear shallow due to the point spread function of the system, discussed below.) There is also a small offset of the fluorescence intensity level due to the fluorescence of the dye along the ordinary axis being nonzero as well as the order parameter of the dye being significantly less than 1 . Analysis of the light collected from the silvered region shows that the data collected from a depth of around $18.6 \mu \mathrm{m}$ and below are largely a reflection of the first half of the cell with the positions of the maxima exhibiting identical spacings. Interestingly, there is a significant increase in the intensity of the reflected light at the liquid crystal/silver interface, which is greater than double the value recorded from the liquid crystal/glass interface. A detailed explanation for this is yet to be determined, but it is believed to be related to the influence of the silver layer on

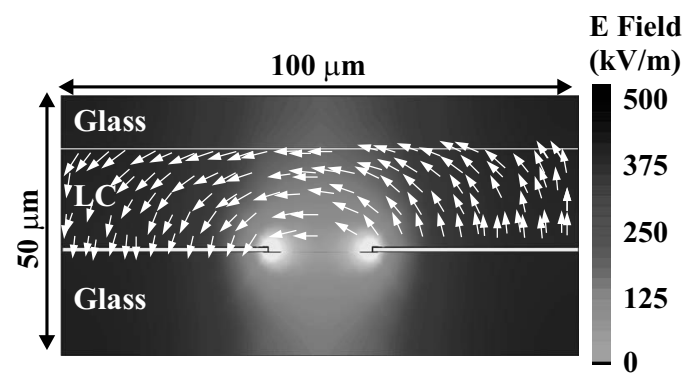

FIG. 6. Modeled electric field for a $25 \mathrm{~V}_{p p}$ in-plane potential difference applied across a $25 \mu \mathrm{m}$ gap. The gray scale indicates the electric field intensity and the arrows indicate the corresponding field lines.

the point-spread function of the system, which is due to the focal point of the system being spread out in the $z$ direction by the optics of the system $[9,10]$. Fluorescence studies using a silvered back plane to enhance backscattered images have previously been used in biological studies using confocal microscopy [11]. It is believed that the enhanced fluorescence intensity produced from the region close to the silver surface may provide a useful additional technique for imaging liquid crystal structures close to an interface.

A 2D model of the stray fields emanating from the edges of the electrode gap in the ZLI-2293 cell is produced by using a finite-element modeling software package (Maxwell $\mathrm{SV}$, Ansoft). The liquid crystal layer is approximated as an isotropic dielectric layer with $\varepsilon=8$ and the entire structure is continuous along the $y$ axis (i.e., end effects are neglected). The result of modeling the fields at an in-plane voltage of $25 \mathrm{~V}_{p p}$ is shown in Fig. 6. It can be seen that the maximum field occurs at the electrode edges with stray fields directed perpendicular to the substrate extending along the $x$ axis, which results in the liquid crystal forming an in-plane cholesteric structure. In contrast, the same fields in the cell containing the negative dielectrically anisotropic material causes the director in this region to remain in the plane of the substrate. The general distribution of the field within the electrode gap agrees well with the regions where the distortion of the cholesteric helix of ZLI-2293 is observed in the FCPM images. The fields are predominantly seen at the edges of the electrode gap, and it is in these same regions that the initial buckling of the cholesteric layers is observed. At higher voltages, the distortion readily extends over more than $20 \mu \mathrm{m}$ along the $x$ axis, and combined with associated elastic energy and surface anchoring considerations the influence of the resulting distortion can extend over an even greater range.

\section{CONCLUSIONS}

This study has shown that FCPM imaging is a powerful technique for imaging liquid crystal structures in 3D. The results of imaging the deformation of cholesteric structures with an applied in-plane field show that the influence of stray fields around the electrode edges extend well beyond the perimeter of the electrode gap, and can be sufficiently strong 
to cause significant reorientations of the liquid crystal outside the intended switching region. With the increasing miniaturization of liquid crystal devices and the use of in-plane switching schemes, the effects of stray fields on adjacent pixels is an important issue and must be addressed when designing such systems.

\section{ACKNOWLEDGMENTS}

The authors would like to acknowledge Dr. G. Zorinyants and S. Cornford from the University of Exeter for useful discussions, and funding from the Engineering and Physical Sciences Research Council, U.K.
[1] M. Ohe and K. Kondo, Appl. Phys. Lett. 67, 3895 (1995).

[2] S. H. Lee, S. L. Lee, and H. Y. Kim, Appl. Phys. Lett. 73, 2881 (1998).

[3] W. M. Liu, J. Kelly, and J. Chen, Jpn. J. Appl. Phys., Part 1 38, 2779 (1999).

[4] I. I. Smalyukh, S. V. Shiyanovskii, and O. D. Lavrentovich, Chem. Phys. Lett. 336, 88 (2001).

[5] I. Dierking, ChemPhysChem 2, 663 (2001).

[6] J. G. White, W. B. Amos, and M. Fordham, J. Cell Biol. 105,
41 (1987).

[7] I. I Smalyukh and O. D. Lavrentovich, Phys. Rev. E 66, 051703 (2002)

[8] B. T. Hallam, Liq. Cryst. 26, 657 (1999).

[9] A. Egner and S. W. Hell, J. Microsc. 193, 244 (1999).

[10] J. B. Pawley, Handbook of Biological Confocal Microscopy (Springer, Berlin, 2006).

[11] C. H. Keith, G. J. Bird, and M. A. Farmer, BioTechniques 25, 858 (1998). 